\title{
Thermal Mechanical Stretching to Imprint Pores Morphology of Nitrocellulose Membrane for Immuno-sensing Application
}

\author{
Mohamad Faizal Khamis \& Siew Chun Low* \\ School of Chemical Engineering, Engineering Campus, Universiti Sains Malaysia, \\ Seri Ampangan, 14300 Nibong Tebal, Pulau Pinang, Malaysia
}

Submitted: 10/1/2020. Revised edition: 24/2/2020. Accepted: 24/2/2020. Available online: 5/3/2020

\begin{abstract}
Performance of membrane such as the lateral flow wicking time and protein binding ability are important to generate consistent results for diagnostics purposes. Different diagnostic kit need different surface properties of membrane, structures and dimension. This work evaluates the feasibility of controlling membrane pores morphology through thermal-mechanical stretching. Results shows that membrane fabricated using longer nitrocellulose (NC) polymer chain length produced smaller pores with lower porosity (56\%). Thus, it took longer time of $32 \mathrm{~s}$ to migrate the testing liquid along the membrane strip. By having higher membrane's porosity $(72.3 \%)$, the membrane synthesized using shorter NC chain length exhibited faster wicking time, which is 3 times faster (wicking time of $8 \mathrm{~s}$ ) than that of the membrane produced with longer NC chain length. In terms of the thermal-mechanical stretching effects, the stretched membranes (both uniaxial and biaxial directions) had demonstrated improved immunoassay performances compared to the unstretched membrane. Specifically, uniaxial stretching is preferable than biaxial stretching configuration, due to the great improvement of lateral wicking time ( $22 \%$ faster) without jeopardize the membrane protein binding capacity (only $1.7 \%$ decrement), in relative to the unstretched membrane. This study provides some interesting insight on the physical membrane modification to provide better performance in immunoassay applications.
\end{abstract}

Keywords: Mechanical stretching, nitrocellulose, immunoassay, membrane, lateral flow

\subsection{INTRODUCTION}

Lateral flow immunoassay tests have experienced remarkable growth in biomedical industry, especially for those with low-resources facilities. In industry, immunoassays are used to detect contaminants in food and water, and in quality control to monitor specific molecules used during product processing [1, 2, 3]. The principal analysis of an immunoassay is based on the specific interactions between a substance of interest (target analyte) and the biological recognition elements (capture analyte such as enzymes, antibodies, nucleic acids, etc.) that are immobilized on the lateral flow membrane [4].

The implementation of lateral flow diagnostics using adsorptive nitrocellulose (NC) membrane as chromatographic media has becoming more apparent $[5,6]$. NC membrane is widely used due to its ability to bind with various antibodies, antigen and other biological components [7]. Besides of its widely usage as pregnancy kit in medical industry, now its application has been expanded to other areas such in detection of viruses $[8,9]$, environmental monitoring [10,

\footnotetext{
* Corresponding to: Siew Chun Low (email: chsclow@usm.my, siewchun@gmail.com) DOI: https://doi.org/10.11113/amst.v24n1.178
} 
11] as well as the detection of contamination in poultry feeds [12]. Comparing to other type of membranes, $\mathrm{NC}$ is preferable to be used in lateral flow mode due its porous structure to easily wick the solution and high binding with biomolecules. Both criteria are important as the target analyte will move parallel along plane of the membrane as liquid moved from one end of the membrane to the other end and bind to the immobilized receptor (capture zone). A pure NC membrane has typical protein binding capacity in the range of $80 \mu \mathrm{g} / \mathrm{cm}^{2}$ to $125 \mu \mathrm{g} / \mathrm{cm}^{2}$ [13].

Lateral flow wicking time and protein binding ability are two important parameters to generate consistent results for diagnostic purposes. Different diagnostic kit need different surface properties of membrane, structures and dimension $[14,15]$. Hence, it is paramount to study the surface and internal layer of the membrane such as its pore size and pore alignment, as it is the fundamental in developing diagnostic kit for medical and healthcare purposes. If the membrane surface and internal layer could be control (imprinted with specific pore structure), various diagnostic kits could be effectively and accurately performed. In fact, the membrane pores structural are commonly controlled by adjusting the casting formulation $[16,17]$ or modify the membrane physically. Physical modification of a membrane is preferable than the complex casting formulation.

Thermal mechanical stretching technique is a technique that applied the mechanical strength and heat treatment to the membrane to yield desired morphology without changing the chemical properties of a membrane $[18,19,20,21]$. The synthesized membrane is stretched under certain heat temperature. The purpose of stretching is to modify the membrane structures, meanwhile, the heat treatment is to ensure the polymer is ductile enough to be stretched. Li and co-workers [22] had carried out a systematic experiment to stretch the high density polyethylene (HDPE) film. In their study, the stretched membrane was shown to have elongated pores and highly distributed along the stretched direction.

The main focus of this work is to produce a highly porous membrane with fast lateral wicking speed (to shorten the diagnostic period) and high protein binding ability (to allow the formation of immunocomplexes on the membrane surface to detect the presence of an analyte in a biological liquid sample). One of the strategy to increase lateral wicking speed of membrane is to enlarge its pore size to allow fast movement of liquid in membrane. However, over-enlarged pores could bring adverse effects to the membrane's protein binding capacity. Hence, this work intend to present the adjustment of the membrane's pore structure through the mechanical stretching at different stretching operating conditions. Although a few previous studies has been conducted on membrane thermal-mechanical stretching [23, 24, 25], most of the studies were focused on single stretching direction and its performances. By such, there is no intensive study on the thermal mechanical stretching on different configurations, such as the biaxial stretching. Technically speaking, the capillary flow rate (lateral wicking speed) is related to the size of the pores oriented in parallel to the wicking direction of the membrane. By having different stretching configurations, the outcome of the lateral wicking flow rate would be different based on the changes orientation of the membrane 
pore size. In addition, the pore shape would also affect the width of the capture reagent line, which in turn defines the width of the signal line when the strip is tested. This work aimed to produce thermalmechanically stretched lateral flow nitrocellulose membrane based on uniaxial and biaxial directions. Membrane morphology and its performances before and after thermalmechanical stretching are compared in this work.

\subsection{METHODS}

\subsection{Materials}

Membranes were casted using short (50-100 kcP) or long (800-1000 kcP) chain $\mathrm{NC}$ and cellulose acetate (CA) polymers from Sigma-Aldrich (Germany). Various solvents (methyl acetate, ethanol), non-solvent (2Propanol) and additive (glycerol) purchased from Merck (Germany) were used to control phase inversion of membrane formation. Bovine serum albumin (BSA) and Ponceau S from Sigma-Aldrich (Germany) were used to quantify the protein binding capacity of membranes.

\subsection{Membrane Preparations}

Unsupported flat sheet NC membranes were prepared using the dry phase inversion method. From the findings of our previous work [26], 4.5 wt.\% NC and $0.5 \mathrm{wt} . \% \mathrm{CA}$ were slowly added to solvent mixture (50 wt.\% methyl acetate and 30 wt.\% ethanol), then stirred at $30{ }^{\circ} \mathrm{C}$ until all polymers dissolved. 2-propanol (10 wt.\%) as non-solvent and additives (2 wt.\% glycerol and 3 wt.\% water) were then added and continued stirred for $5 \mathrm{~h}$. The dope solution was casted on a glass plate using Elcometer thin film applicator with depth of the casting of $700 \mu \mathrm{m}$. The casted membrane was then dried in a vacuum oven overnight at $35{ }^{\circ} \mathrm{C}$. Surface morphology of the membrane was analyzed using scanning electron microscope (Hitachi TM-3000, Japan) at an accelerating voltage of $3 \mathrm{kV}$. Bonding characterization of the membrane surface was confirmed using Thermo Scientific fourier transform infrared spectrometer (NICOLET iS10, USA), fixed at the incidence angle of $45^{\circ}$, from $500 \mathrm{~cm}^{-1}$ to $4000 \mathrm{~cm}^{-1}$ wavenumber. Pore characteristics of the membrane was analyzed using gasliquid porometer (Porolux 1000, Belgium) within pressure range of 0-6 bars. By measuring the pressure and corresponding flow at every increasing pressure step, the wet- and dry-flow curves for the membrane were plotted. These curves were used to calculate the pore characteristics of the membrane. Relationship between the membrane pore size and the corresponding pressure is expressed by the Young-Laplace equation. In this work, porosity $(\varepsilon)$ of the membrane was calculated based on:

$$
\varepsilon=\frac{V_{A}-V_{E}}{V_{A}} \times 100
$$

$\mathrm{V}_{\mathrm{A}}$ (apparent volume) is determined based on the film thickness and the surface area $(2 \mathrm{~cm} \quad x \quad 2 \mathrm{~cm}) . V_{E}$ (existent volume) is calculated using the corresponding polymer density $\left(\mathrm{NC}=1.23 \mathrm{~g} / \mathrm{cm}^{3}\right.$ and $\mathrm{CA}=1.28$ $\mathrm{g} / \mathrm{cm}^{3}$ ) and dry weight of the membrane.

\subsection{Thermal-mechanical Stretching}

Membrane was stretched using thermal-mechanical stretching technique with uniaxial $(100 \mathrm{~mm} \times 60$ $\mathrm{mm}$ membrane size) and biaxial (60 $\mathrm{mm}$ x $60 \mathrm{~mm}$ membrane size) stretching configurations, as shown in 
Figure 1. Membrane sample was gripped by a rigid support beam on the stretching platform in heat insulated chamber. It was then heated up to 50 ${ }^{\circ} \mathrm{C}$ and left in the heat insulated chamber for 10 minutes, to ensure the polymer is ductile enough to be significantly stretched. The stretching rate was controlled by an electric stepper motor (Vexta EM569-NA, Japan) with a stepping controller (Vexta UDX 5107N). In this study, membrane sample was stretched to $10 \%$ of the elongation length and at stretching speed of $0.07 \mathrm{~m} / \mathrm{s}$. In order to maintain the membrane structure after stretched, the membrane sample was held under tension for $15 \mathrm{~min}$ and then air-cooled to room temperature before removed from the stretching machine. The membrane elongation \% was calculated based on:

$$
\text { Elongation, } \%=\frac{l_{s}-l_{o}}{l_{s}} \times 100
$$

where $l_{o}$ and $l_{s}$ are refers to the length of the membrane before and after stretched $(\mathrm{mm})$.

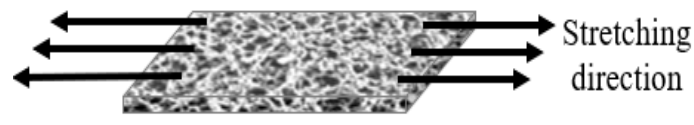

Uni-axial stretching

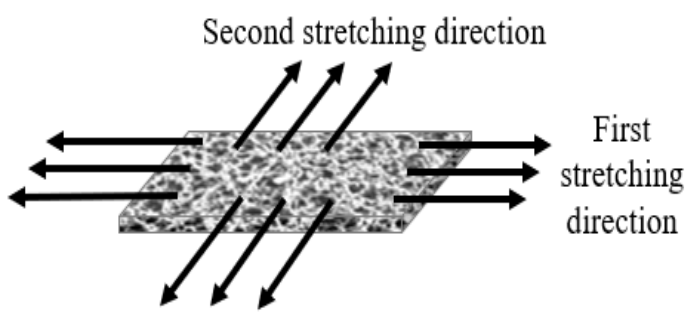

Bi-axial stretching

Figure 1 Uniaxial and biaxial stretching of membrane

\subsection{Evaluation of the Membrane Performance as an immunoassay}

Membrane binding ability was measured using $10 \mathrm{~mm} \mathrm{x} 10 \mathrm{~mm}$ samples. First, membranes samples were incubated in $3 \mathrm{~mL}$ of BSA solution with $0.05 \mathrm{M}$ phosphate buffer $(\mathrm{pH} 7.0,3 \mathrm{mg} / \mathrm{mL})$ and shaken for $3 \mathrm{~h}$ at $25^{\circ} \mathrm{C}$, as described in the previous work [27]. Then, the membrane samples were washed (repeated twice) with phosphate buffer to remove unbound BSA. Subsequently, samples were transferred into test tubes and 2 $\mathrm{mL}$ of bicinchoninic acid working reagent (Merck, Germany) were added. Test tubes were incubated at $37{ }^{\circ} \mathrm{C}$ for $30 \mathrm{~min}$, where the bicinchoninic acid working reagent would reacts with BSA bound on the membrane surface, to change the solution color from green to purple, which is in proportion to the protein concentration.

BSA concentration was detected at $562 \mathrm{~nm}$ wavelength using a spectrophotometer (Spectronic Genesys, USA). Using the preliminarily plotted standard curve, the corrected absorbance readings for the samples were interpolated.

To monitor the spreading of protein onto membrane, the dot staining technique with Ponceua S was applied. $1 \mu \mathrm{L}$ of BSA was first spotted onto the surfaces of membranes and then left to air-dried. The sample was then immersed in a sufficient amount of Ponceau $\mathrm{S}$ solution for 5 minutes to stain the immobilized BSA, then rinsed twice with DI water to wash off the undesired membrane background. Stained protein with Ponceau S were scanned using Heiland Electronics $\mathrm{GmbH}$ densitometer (TRD 4, Germany) at the scanning wavelength of $560 \mathrm{~nm}$ in reflectance mode, with a measuring aperture diameter of $3 \mathrm{~mm}$. The color density of the stained protein 
spot were measured. Each value represents an average value of six spots.

The liquid flow time (wicking time) measurement was conducted on $2 \mathrm{~cm}$ wide and $5 \mathrm{~cm}$ long of the membrane strips cut from the synthesized NC membranes, with distilled water was used as the wicking solution. Experiment was conducted at room temperature $\left(30{ }^{\circ} \mathrm{C}\right)$, with one end of membrane strip $(5 \mathrm{~mm})$ was dipped into the wicking solution. Time measurement was started when the wicking solution was started to migrate along the membrane strip (parallel along the membrane plane) until it reached $2 \mathrm{~cm}$ and $3 \mathrm{~cm}$ height of the membrane.

\subsection{RESULTS AND DISCUSSION}

As for an immunoassay, a known capture reagent is first immobilized at the predetermined target analyte capture region (test and control lines), as shown in Figure 2. Subsequently, the test sample containing target analyte is absorbed by the sample pad through capillary forces to the conjugate pad, where preloaded recognition element is imbedded.
Typical fluid sample such as serum, blood, urine or saliva can be tested. As the solution (fluid sample together with the recognition element) flows along the membrane to reach the test and control line, the target analyte would bind with the pairing immobilized capture reagent $[6,28]$. The purpose of membrane is to bind the capture reagent at the test and control lines; and also to maintain lateral capillary flow for the target analyte to move fast within the porous structure to reach to the test and control lines [29]. Without the membrane, the diagnostic test strip would never be able to function. Membrane with large pore structure would contribute to low protein immobilization but fast lateral wicking speed. In contrast, small pores membrane provides high binding sites to immobilize the capture reagent, but at the same time, it also causes high resistance for the testing liquid to laterally flow along the membrane strip. Based on this working principle, the synthesized membrane in this work were evaluated based on two parameters, i.e. membrane's protein binding capacity and lateral wicking time for the testing solution to migrate $2 \mathrm{~cm}$ and $3 \mathrm{~cm}$ along the membrane strip.

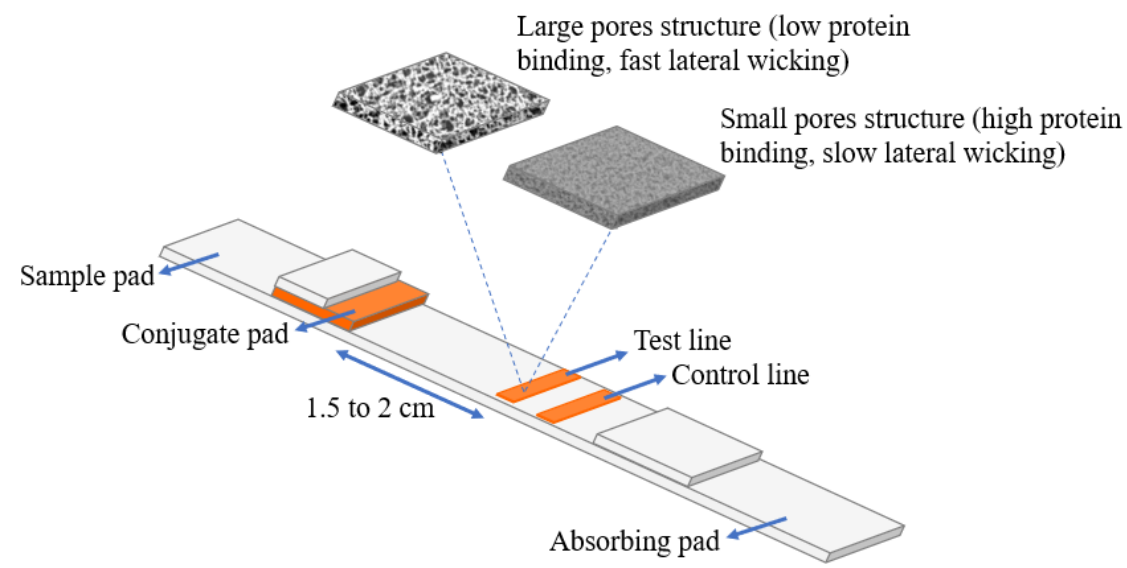

Figure 2 Schematic of lateral flow immunoassay 
Polymer chain length plays an important role to produce membrane with different pore characteristics and thus it's performance in bio-sensing application. ATR-FTIR spectra of membranes produced using short (50$100 \mathrm{kcP})$ and long (800-1000 kcP) NC polymer chain length is shown in Figure 3a. Overall, FTIR spectra for the membrane with longer polymer chain length had demonstrated higher transmittance intensity. Stronger peak intensity at $1640 \mathrm{~cm}^{-1}$ was corresponded to the symmetric stretching band of $\mathrm{ONO}_{2}$ groups, which confirm the presence of nitrate group in the synthesized membrane [30]. The other absorption band that relate to nitrate group were $\mathrm{v}_{\mathrm{s}}-\mathrm{NO}_{2}$ at $1275 \mathrm{~cm}^{-1}$ and $\mathrm{v}-\mathrm{NO}$ at $833 \mathrm{~cm}^{-1}$ [31]. Higher polymer chain length contained more nitrate groups, which expect to have more active sites to interact with biomolecules (capture reagent) that immobilize onto the membrane surface. Hence, increase the sensitivity of the bio-sensing application.

\section{(a)}

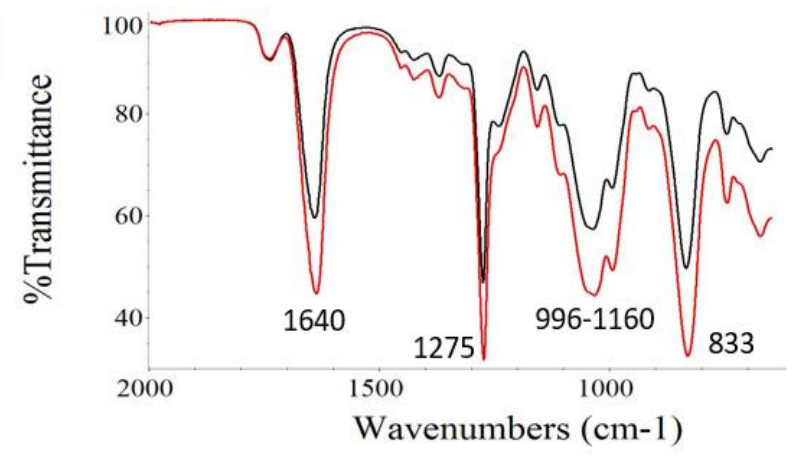

(b)

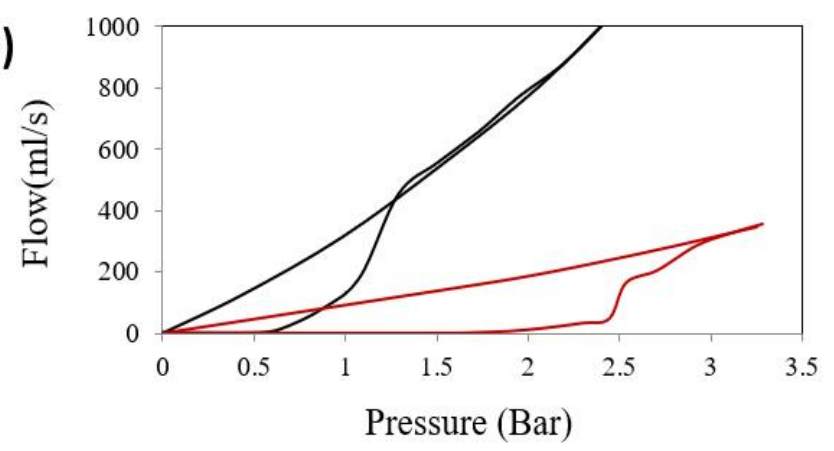

(c)

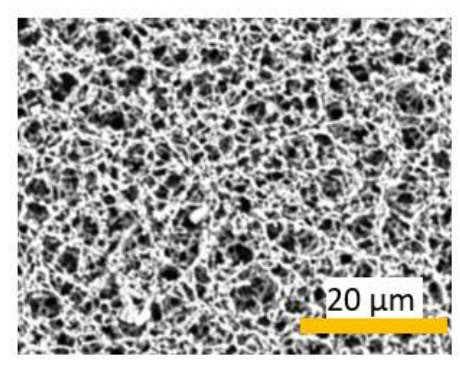

Short chain nitrocellulose

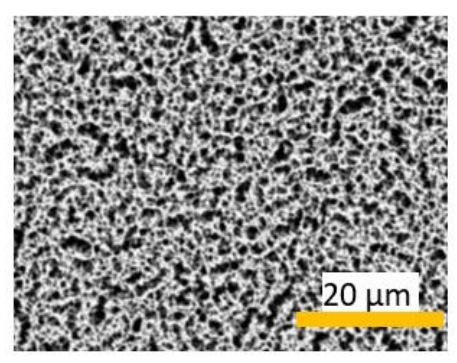

Long chain nitrocellulose

Figure 3 Nitrocellulose membranes produced with short (black line, 50-100 kcP) and long (red line, 800-1000 kcP) polymer chain length: (a) FTIR spectrum (b) membrane pores characteristic (c) SEM images for membrane surface topography

When analyzing the pores characteristics of the membranes using porometer, at a specific pressure (Figure 3b), the gas flow rate through a wet sample is always smaller than that flow through the corresponding dry sample until finally the two curves meet together [16]. At this meeting point, all the wetting liquid in the through-pores have move out from the sample due to the higher exerted pressure. The short chain NC membrane (50-100 $\mathrm{kcP}$ ) required about 1.2 bar of pressure to purge out all liquid, while the long chain NC membrane (800-1000 $\mathrm{kcP}$ ) required about 2.9 bar to empty the entire pores. In consistent to the SEM images (Figure 3c), the long chain NC membrane has demonstrated smaller 
pores distribution, thus, higher pressure needed to purge out the wetting liquid. During the measurement (Figure 3b), as the gas pressure increased, at specific point the first pore was emptied. This is the pore with the largest through-pore diameter. In this work, bubble point was found at $0.852 \mu \mathrm{m}$ and $0.287 \mu \mathrm{m}$ with corresponded bubble point pressure of 0.5371 bar and 1.607 bar for both short and long chain NC membranes, respectively. Membrane with long NC chain length showed narrower range of pore distribution within 0.1-0.29 $\mu \mathrm{m}$, while membrane produced using short NC polymer chain had demonstrated broader range of pore distribution within $0.30-0.85 \mu \mathrm{m}$.

These porometer analyzed data were in-lined with the observation from SEM images (Figure 3c). During dry phase inversion, the polymer distribution at the onset of precipitation provided a higher polymer density near to the membrane-air interface. At higher polymer viscosity (longer NC polymer chain length), fast phase inversion occurred at the membrane outer layer and slow evaporation rate in the inner layer caused high density of solid polymer to be formed at the surface of membrane. Thus, greater interfacial stresses were produced between the polymer matrixes. As a consequence, smaller pores were formed at the membrane surface.

Fast lateral flow wicking time and high membrane's protein binding capacity are two important parameters to generate consistent results for diagnostics purposes. Fast lateral wicking membrane is favorable due its ability to easily move the solution to the detection zone, while high membrane protein binding capacity ensure sensitive capture of analyte. As expected, the smaller the pores structure of the membrane, the higher the color density measured by densitometer. Color densities of 0.09 and 0.14 were obtained for membranes produced by short and long chain NC polymer, respectively. As discussed, the casted film with longer polymer chain would formed a membrane with smaller pores. Since the protein molecules were immobilized onto the membrane surface, the smaller pores would reduce the protein diffusivity and accumulated at smaller and enclosed area. In turns, higher color density was observed.

Results in Figure 4 showed that the membrane's porosity was directly related to the decrease of wicking time (faster of DI water to migrate $2 \mathrm{~cm}$ height along the membrane strip). Membrane produced using longer NC polymer chain length took longer time to migrate along the membrane strip. By having higher membrane's porosity (72.3\%), membrane with shorter polymer chain length (50-100 kcP) exhibited faster wicking time of $8.1 \mathrm{~s}$, which is 3 times faster than the membrane with longer chain polymer (wicking time of $31.7 \mathrm{~s}$ and membrane porosity of $56 \%$ ). Based on this results, the short chain polymer $(50-100 \mathrm{kcP})$ is preferable to produce an immunomembrane, due to its faster wicking speed and comparable binding capacity to that of longer chain polymer (800$1000 \mathrm{kcP}$ ).

Due to the gravity effect, wicking time from one point to another point on the membrane strip with the same distance was not proportional. Longer wicking time was needed with an increase in the migration distance of the DI water in membrane strip. As shown in Figure 4, the deionized water took $8.1 \mathrm{~s}$ to migrate $2 \mathrm{~cm}$. On the same membrane strip, the deionized water took $17.3 \mathrm{~s}$ to migrate $3 \mathrm{~cm}$ in height on the membrane strip. In some tests, diagnostic kit is designed to support in a vertical position, for example, mounted in a 96-well plate. 
Thus, the gravitational effects could somehow affect the testing duration if the capture zone (testing line) is designed with a longer distance from the sampling pad.

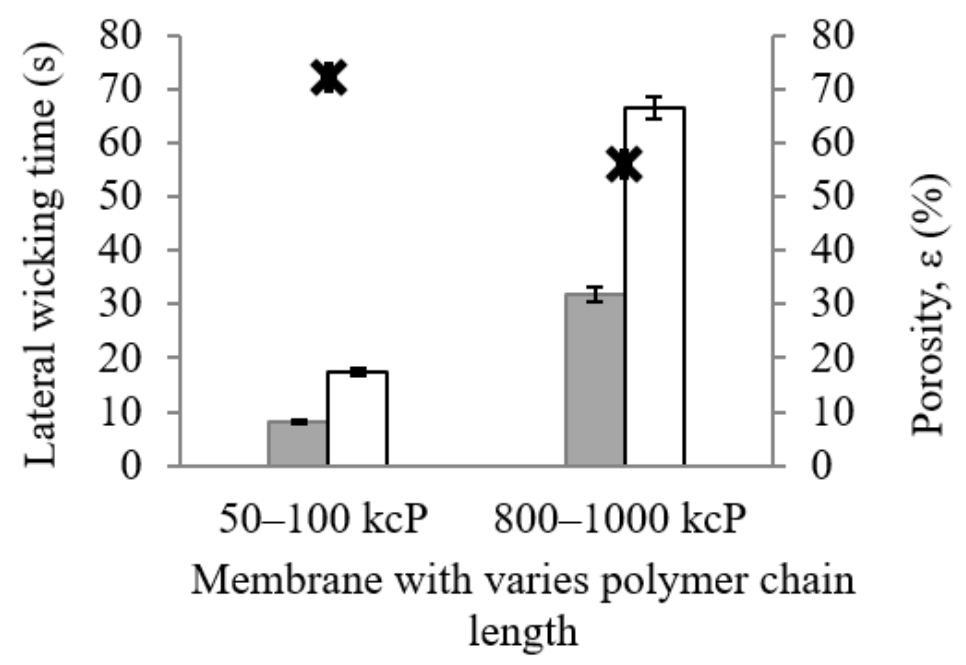

$\square$ To migrate $2 \mathrm{~cm} \square$ To migrate $3 \mathrm{~cm} \mathbf{x}$ Porosity

Figure 4 Lateral wicking performance and porosity of membrane prepared using short (low viscosity, 50-100 kcP) and long (high viscosity, 800-1000 kcP) polymer length

This study proposed to use thermal mechanical stretching to elongate the membrane to desire pore morphology without changing its the chemical properties. When the membranes were casted at different thickness, it provides different mechanical strength to the membrane $[32 ; 33]$. Figure 5a shows the relationship of the membrane thickness with its respective maximum elongation. As shown, the maximum elongation did not show proportional to the thickness of the membrane. The elongated ability was possessed at higher rate for membrane with thickness of 110-150 $\mu \mathrm{m}$. For membrane thicker than $150 \mu \mathrm{m}$, the effects of membrane elongation has reduced, which would defeat the purpose of thermal mechanical treatment on the membrane. Thus, in this work, membranes were produced at thickness of ca. $120 \mu \mathrm{m}$.

Figure $5 b$ shows the image of membrane after stretched. When uniaxial-stretching was applied to the membrane (stretched on left and right end of the membrane strip), the stretching forces have distributed within the sample. However, the center region would be affected more. Thus, curve shape formation was observed. However, because of only $8 \%$ elongation was applied, the shape of the protein dot stained with Ponceau $S$ (red color dots) remained spherical and do not demonstrate obvious elongated shape as expected.

Further analysis was carried out using SEM. As shown (Figure 6), similar surface pore structures were observed for membranes before stretched and after uniaxial and biaxial stretched. The pore elongation due to stretching effects was not obvious. As discussed, this might because of only $8 \%$ elongation was applied, where the stretching forces have eventually shared by all pore matrices during the thermal-mechanical stretching. When the stretching was performed more than $10 \%$, the membrane was broken into two pieces due to the fragile characteristics of the NC polymer. 


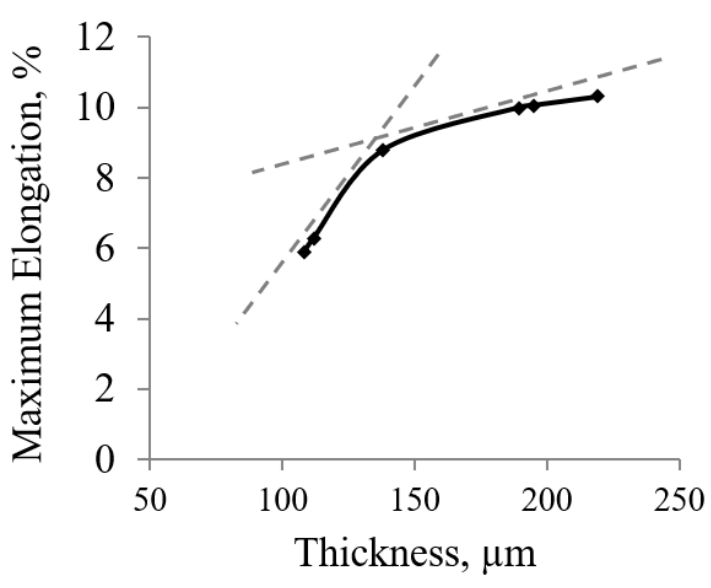

(a)

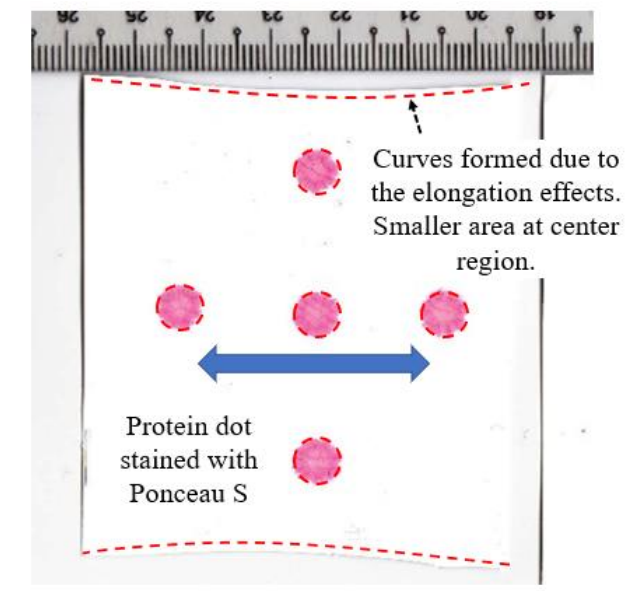

(b)

Figure 5 Effects of membrane elongation: (a) maximum elongated length in different membrane thickness. (b) stretched membrane with uniaxial configuration (double end arrow shows the stretching direction, stretching condition: temperature $55{ }^{\circ} \mathrm{C}$, speed: $0.01 \mathrm{~mm} / \mathrm{s}$, elongation length: $8 \%$ )

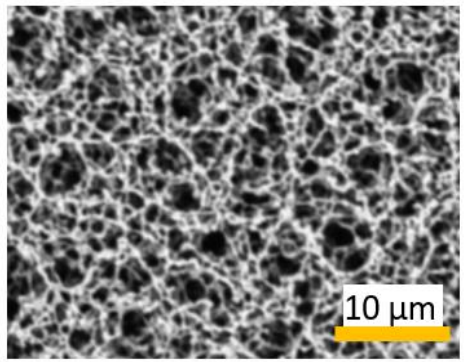

(a)

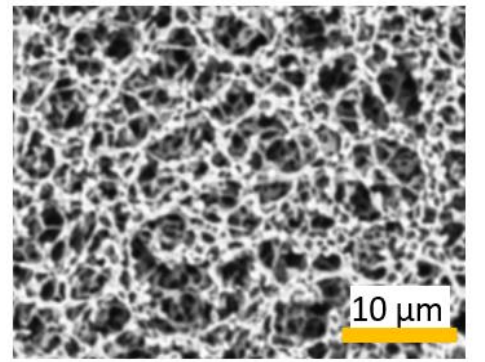

(b)

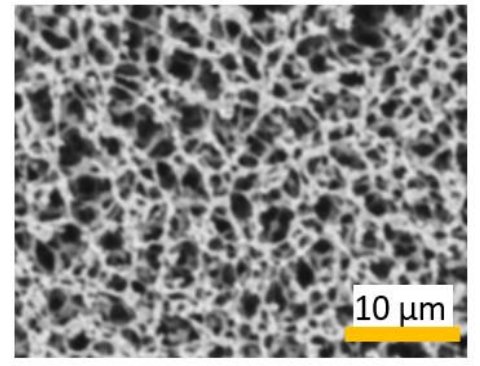

(c)

Figure 6 SEM surface images of (a) unstretched (b) uniaxial-stretched (c) biaxial-stretched membranes

Stretched membranes (uniaxial and biaxial) were also analyzed based on its performances on the lateral wicking time and protein binding capacity. As shown (Figure 7a), membranes treated with thermal-mechanical stretching operations had demonstrated faster lateral wicking speed or shorten lateral wicking time to migrate testing solution towards the protein capture zone of a membrane strip. Membrane with uniaxial stretched showed the fastest wicking time, took $6.3 \mathrm{~s}$ to migrate $2 \mathrm{~cm}$ and $13.3 \mathrm{~s}$ to migrate 3 $\mathrm{cm}$ along the membrane strip, which are $22 \%$ and $23 \%$ faster compared to the unstretched membrane (lateral wicking time of $7.9 \mathrm{~s}$ and $16.9 \mathrm{~s}$ to migrate $2 \mathrm{~cm}$ and $3 \mathrm{~cm}$, respectively). This is due to the aligned pores after thermal-mechanical stretched that reduced the flow resistance when the testing fluid was migrated along the membrane strip. When the membrane was stretched in biaxial direction, the overall pores were eventually enlarged, although not significant to be observed in SEM images (Figure 6). Thus, a relatively faster lateral wicking speed with shorter time was also observed, i.e. $6.6 \mathrm{~s}$ of wicking time to migrate 2 $\mathrm{cm}$ along the membrane strip or $17 \%$ faster than the unstretched membrane. 


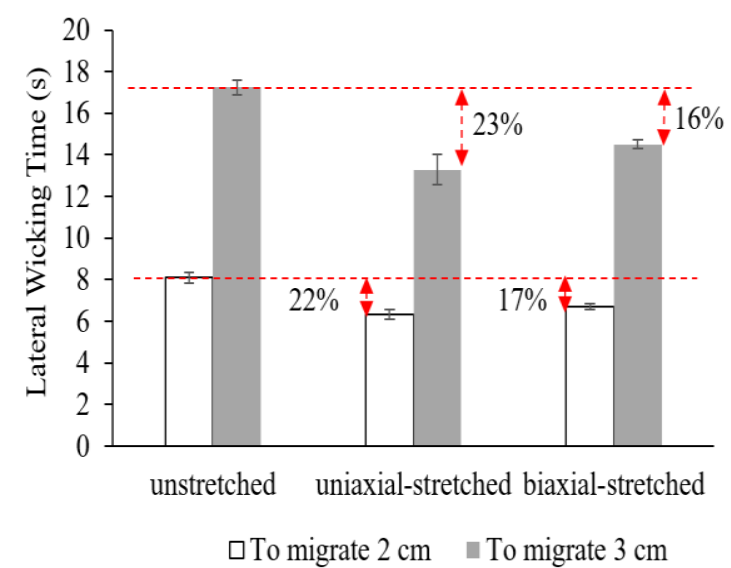

(a)

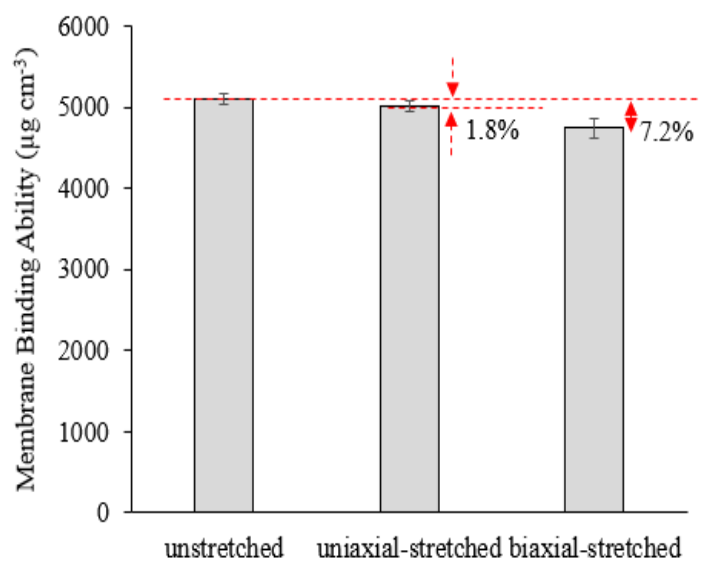

(b)

Figure 7 Performances of membranes before and after stretching (a) lateral wicking time (stretching condition: temperature $55{ }^{\circ} \mathrm{C}$, speed: $0.01 \mathrm{~mm} / \mathrm{s}$, elongation length: $8 \%$ ) (b) protein binding capacity (using bovine serum albumin as model protein)

Membrane's protein ability is another parameter that determine the efficiency of an immunoassay. Primarily, protein binding is influence by the pore size of the membrane. Figure $7 \mathrm{~b}$ shows the protein binding ability of the membrane before and after thermal-mechanically stretched. The unstretched membrane had demonstrated the highest protein binding ability at $5107 \pm 68 \mu \mathrm{g} \mathrm{cm}^{-3}$. By thermal-mechanically modified, membranes underwent uniaxial and biaxial stretching had evidenced slightly lower protein binding, at $5017 \pm 63 \mu \mathrm{g} \mathrm{cm}^{-3}$ and $4741 \pm 114 \mu \mathrm{g}$ $\mathrm{cm}^{-3}$, respectively. Larger pores and porosity of the stretched membrane would decrease the total surface area for electrostatic binding between protein molecules and membrane. However, the adverse effects of thermal-mechanical stretching on the membrane's protein binding ability is not significant, less compared to the enhancement for the lateral wicking test. Only $1.8 \%$ and $7.2 \%$ decrement of protein binding for uniaxial and biaxial stretched membrane. From both results of lateral wicking time and protein binding, enhanced of lateral wicking (up to $23 \%$ improvement) was much significant than that of decrement of the protein binding (down to $7.2 \%$ ).

\subsection{CONCLUSION}

In summary, the shorter chain polymer $(50-100 \mathrm{kcP})$ is preferable to produce an immuno-membrane, due to its faster wicking speed and comparable binding capacity than that of longer chain polymer $(800-1000 \mathrm{kcP})$. In term of different stretching configurations, the uniaxial stretching strategy was better than that of biaxial, with better enhancement of lateral wicking speed and smaller decrement of protein binding. Most importantly, both stretched membrane showed better immunoassay performances than the unstretched membrane. Hence, the proposed thermal-mechanical stretching in this work is still consider efficient to improve the immunoassay performance of a membrane.

\section{ACKNOWLEDGEMENT}

This work is supported by Bridging Grant Universiti Sains Malaysia 304.PJKIMIA.6316534 


\section{REFERENCES}

[1] Yahaya, M. L., Zakaria, N. D., Noordin, R., and Razak, K. A. 2019. The Effect of Nitrocellulose Membrane Pore Size of Lateral Flow Immunoassay on Sensitivity for Detection of Shigella sp. in milk sample. Materials Today: Proceedings. 17: 878-883.

[2] Zhang, C., Han, Y., Lin, L., Deng, N., Chen, B., and Liu, Y. 2017. Development of Quantum Dots-labeled Antibody Fluorescence Immunoassays for the Detection of Morphine. Journal of Agricultural and Food Chemistry. 65: 1290-1295.

[3] Azmi, N. A., Ahmad, S. H., and Low, S. C. 2018. Detection of Mercury Ions in Water Using a Membrane-based Colorimetric Sensor. RSC Advances. 8: 251261.

[4] Shaimi, R., Ketar Mokhtar Nabilah M., Tan, P. C., Jawad Zeinab, A., and Low, S. C. 2016. Chemical Oxidative Polymerization of Conductive Polyaniline-iron Oxide Composite as an ElectroTransducer for Electrochemical Sensing Applications. $e$ Polymers. 16: 225.

[5] Hegener, M. A., Li H., Han, D., Steckl, A. J., and Pauletti, G. M. 2017. Point-of-care Coagulation Monitoring: First Clinical Experience Using a Paper-based Lateral Flow Diagnostic Device. Biomedical Microdevices. 19: 64.

[6] Qian, S., and Bau, H. H. 2004. Analysis of Lateral Flow Biodetectors: Competitive Format. Analytical Biochemistry. 326: 211-224.

[7] Low, S. C., Shaimi, R., Thandaithabany, Y., Lim, J. K., Ahmad, A. L., and Ismail, A.
2013.

Electrophoretic

Interactions between

Nitrocellulose Membranes and Proteins: Biointerface Analysis and Protein Adhesion Properties. Colloids and Surfaces B: Biointerfaces. 110: 248-253.

[8] Xiao, M., Xie K., Dong X., Wang L., Huang C., Xu F., Xiao W., Jin M., Huang B., and Tang Y. 2019. Ultrasensitive Detection of Avian Influenza A (H7N9) Virus Using Surface-enhanced Raman Scattering-based Lateral Flow Immunoassay Strips. Analytica Chimica Acta. 1053: 139-147.

[9] Kumar, S., Bhushan, P., Krishna, V., and Bhattacharya, S. 2018. Tapered Lateral Flow Immunoassay Based Point-ofcare Diagnostic Device for Ultrasensitive Colorimetric Detection of Dengue NS1. Biomicrofluidics. 12: 034104

[10] Xing, C., Liu, L., Song, S., Feng, M., Kuang, H., and Xu, C. 2015. Ultrasensitive

Immunochromatographic Assay for the Simultaneous Detection of Five Chemicals in Drinking Water. Biosensors and Bioelectronics. 66: 445-453.

[11] Wang, X., Sun J., Tong J., Guan X., Bian C., and Xia S. 2018. Paper-based Sensor Chip for Heavy Metal Ion Detection by SWSV. Micromachines. 9.

[12] Lin, C. W., Kuo, J. C., Liu, H. L., Cheng, Y. S., and Huang, H. L. 2018. A New Method for Detection of Single Nucleotide Polymorphism in a Female Reproduction-associated Gene, Tmigd1, of Anas Platyrhynchos Using a Strip Biosensor with Gold Nanoparticles. Poultry Science. 97: 3456-3462.

[13] Harvey, B. M., and Soundy, P. 2008. Southern Blotting as a 
Diagnostic Method. Molecular Biomethods Handbook. Second Edition.

[14] Shaimi, R., and Low, S. C. 2018. Morphological Characteristics of Polymeric Nylon-6 Film as Biological Recognition Interface for Electrochemical Immunosensor Application. Journal of Applied Polymer Science. 135: 46741.

[15] Azmi, N. A., and Low, S. C. 2017. Investigating Film Structure of Membrane-based Colorimetric Sensor for Heavy Metal Detection. Journal of Water Process Engineering. 15: 37-42.

[16] Low, S. C., Ahmad, A. L., Ideris, N., and Ng, Q. H. 2012. Interaction of Isothermal Phase Inversion and Membrane Formulation for Pathogens Detection in Water. Bioresource Technology. 113: 219-224.

[17] Ahmad, A. L., Low, S. C., Shukor, S. R. A., and Ismail, A. 2008. Synthesis and Characterization of Polymeric Nitrocellulose Membranes: Influence of Additives and Pore Formers on the Membrane Morphology. Journal of Applied Polymer Science. 108: 25502557.

[18] Ahmad, A. L., Low, S. C., Abd Shukor, S. R., and Ismail, A. 2009. Morphological and Thermal-mechanical Stretching Properties on Polymeric Lateral Flow Nitrocellulose Membrane. Industrial and Engineering Chemistry Research. 48: 34173424.

[19] Fereydoon, M., Tabatabaei, S. H., and Ajji, A. 2015. Effect of Uniaxial Stretching on Thermal, Oxygen Barrier, and Mechanical Properties of Polyamide 6 and Poly(m-xylene adipamide)
Nanocomposite Films. Polymer Engineering and Science. 55: 1113-1127.

[20] Komaladewi, A. A. I. A. S., Anisah, S., Wardani, A. K., Surata, I. W., Subagia, I. D. G. A., and Wenten, I. G. 2019. The Effect of Annealing and Stretching Parameters on the Structure and Performance of Polypropylene Hollow Fiber Membrane. Materials Research Express. 6: 054001.

[21] Choi, W., Chun K. Y., Kim, J., and Han, C. S. 2017. Ion Transport through Thermally Reduced and Mechanically Stretched Graphene Oxide Membrane. Carbon. 114: $377-$ 382.

[22] Li, X., Lin, Y., Su, F., Chen, X., Zhang, W., Lv, F., Meng, L., Zhang, Q., and Li, L. 2017. Mechanical Energy and Thermal Effect Controlled Micropore Nucleation and Growth Mechanism in Oriented High Density Polyethylene. Polymer. 133: 240-249.

[23] Ahmad, A. L., Low, S. C., Shukor, S. R. A., and Ismail, A. 2009. Optimization of Membrane Performance by Thermalmechanical Stretching Process Using Responses Surface Methodology (RSM). Separation and Purification Technology. 66: 177-186.

[24] Mahmoudi, F., Saljoughi, E., and Mousavi, S. M. 2013. Promotion of Polysulfone Membrane by Thermal-mechanical Stretching Process. Journal of Polymer Research. 20: 96.

[25] Li, C., Liu, J., Guan, R., Zhang, P., and Zhang, Q. 2007. Effect of Heating and Stretching Membrane on Ionic Conductivity of Sulfonated Poly(phenylene 
Oxide). Journal of Membrane

Science. 287: 180-186.

[26] Ahmad, A. L., Low, S. C., Abd Shukor, S. R., and Ismail, A. 2007. Preparation and Characterization of Various Membrane Morphologies for Lateral Flow Immunoassay Development. Journal of Applied Membrane Science \& Technology. 5: 23-29.

[27] Shaimi, R., and Low, S. C. 2016. Prolonged Protein Immobilization of Biosensor by Chemically Cross-linked Glutaraldehyde on Mixed Cellulose Membrane. Journal of Polymer Engineering. 36: 655661.

[28] Ang, Q. Y., and Low, S. C. 2019. Feasibility Study on Molecularly Imprinted Assays for Biomedical Diagnostics. Sensor Review. 39: 862-873.

[29] Ahmad, A. L., Low, S. C., Shukor, S. R. A., Fernando W. J. N., and Ismail, A. 2010. Hindered Diffusion in Lateral Flow Nitrocellulose Membrane: Experimental and Modeling Studies. Journal of Membrane Science. 357: 178-184.

[30] Gelaw, T. K., Güell, C., Ferrando, M., and De Lamo-Castellví, S. 2014. Use of Attenuated Total
Reflectance Infrared Microspectroscopy Combined with Multivariate Analysis to Study Membrane Fouling. Journal of Food Engineering. 143: 69-73.

[31] Su, X., Zhao, Q., Zhang, D., and Dong, W. 2015. Synthesis and Membrane Performance Characterization of Selfemulsified Waterborne Nitrocellulose Dispersion Modified with Castor Oil. Applied Surface Science. 356: 610-614.

[32] Ahmad, A. L., Low S. C., and Shukor S. R. A. 2007. Effects of Membrane Cast Thickness on Controlling the Macrovoid Structure in Lateral Flow Nitrocellulose Membrane and Determination of Its Characteristics. Scripta Materialia. 57: 743-746.

[33] Ghasemi, S. M., and Mohammadi, N. 2014. The Trend of Membrane Structure Evolution Under Shear and/or Elongation Flow Fields of Immersion Precipitated Spun Tapes. Journal of Membrane Science 460: 185-198. 